INT-PUB 04-01

\title{
Dynamic universality class of the QCD critical point
}

\author{
D. T. Son ${ }^{1, *}$ and M. A. Stephanov ${ }^{2,3, \dagger}$ \\ ${ }^{1}$ Institute for Nuclear Theory, University of Washington, Seattle, Washington 98195-1550 \\ ${ }^{2}$ Department of Physics, University of Illinois, Chicago, Illinois 60607-7059 \\ ${ }^{3}$ RIKEN-BNL Research Center, Brookhaven National Laboratory, Upton, New York 11973
}

(Dated: January 2004)

\begin{abstract}
We show that the dynamic universality class of the QCD critical point is that of model $\mathrm{H}$ and discuss the dynamic critical exponents. We show that the baryon diffusion rate vanishes at the critical point. The dynamic critical index $z$ is close to 3 .
\end{abstract}

*Electronic address: son@phys.washington.edu

${ }^{\dagger}$ Electronic address: misha@uic.edu 


\section{INTRODUCTION}

The phase diagram of QCD is a focus of many theoretical investigations [1]. Heavy-ion collision experiments can probe a certain part of this phase diagram. The temperature and the baryon chemical potential at freezeout are determined, using statistical model fits to particle yields and spectra, to span the domain $T \sim 100-180 \mathrm{MeV}$ and $\mu_{\mathrm{B}}=50-600 \mathrm{MeV}$ 2]. This is the region where the critical point of QCD, as strongly suggested by model calculations [3] and lattice Monte Carlo results [4], is located. The critical point is an end point of a first order phase transition line separating, in the chiral limit, the chirally broken and chirally symmetric phases. The precise location of the critical point is still unknown.

Experimental signatures, based on the singular behavior of thermodynamic functions near the critical point, were suggested in Refs. [5, 6]. The characteristic feature of all such signatures is the non-monotonic dependence on the value of experimentally controlled parameter, such as $\sqrt{s}$, as the critical point is approached and passed. An attempt to estimate the effects of critical dynamics on experimental observables was made in Ref. [6], where it has been shown that the effects can easily exceed the experimental background by orders of magnitude. The major limiting factor is the finite size and time effects, which round up the critical singularity in all observables.

Near criticality, the crucial quantity is the value of the largest correlation length $\xi$. For example, the singular contributions to event-by-event fluctuation observables studied in Ref. [6] are proportional to $\xi^{2}$. The divergence of $\xi$ is limited by two effects: i) the finite system size, $\mathcal{O}(10 \mathrm{fm})$ for heavy-ion collisions, and ii) the finite evolution time. As pointed out in Refs. [6, 7], the second effect is more important. Indeed, the time during which the the correlation length reaches its equilibrium value diverges as $\tau \sim \xi^{z}$, which defines the dynamic scaling exponent $z$. The finite evolution time limits the correlation length to be $\xi<(\text { time })^{1 / z}$. Since $z>1$ and typical evolution times of the heavy ion collisions are of the same order as the spatial size, the time limit is more stringent then spatial size limitation $\xi<$ (size). The value of $z$ depends on the dynamic universality class [8] of the critical point.

The purpose of this paper is to determine this universality class. While the static universality class is beyond doubt that of the $3 \mathrm{~d}$ Ising model, the question about the dynamic universality class has not been satisfactorily answered in the literature. Some arguments were given in Ref. [6] that the universality class could be that of model $\mathrm{H}$ in Hohenberg and Halperin's classification [8], - a model of the liquid-gas phase transition. The question was revisited in Ref. [7], where it was argued that the universality class is that of model C. The problem is not trivial because of an additional mode compared to the ordinary liquid-gas phase transition — the QCD chiral condensate $\bar{q}$.

We shall see that mixing between the chiral condensate $\bar{q} q$ and the baryon density $n$ leaves out only one truly hydrodynamic mode - a linear combination of the two. The hydrodynamic mode is conserved and couples to the energy-momentum density. Consequently, the relevant hydrodynamic modes near the critical point are the same as in the model $\mathrm{H}$. Thus we predict $z \approx 3$.

Our results are in qualitative agreement with the arguments of Fujii based on a recent study 9]. The study [9] employed model calculations, and did not, in particular, take into 
account the coupling to energy-momentum density. Our approach is model-independent and is based on the straightforward application of the hydrodynamic theory, reviewed, e.g., in Ref. [10].

The paper is organized as follows. In Sec. III we identify the set of hydrodynamic modes near the critical point. In Sec. III we discuss a simplified problem, where we forcibly neglect the energy-momentum density. The purpose is to demonstrate that only one linear combination of the chiral order parameter $\langle\bar{q} q\rangle$ and the baryon number density $n$ is a true hydrodynamic mode. To keep the discussion simple, we shall work at the mean field level, sufficient to identify the correct universality class. We refer to the literature for going beyond mean field. In Sec. IV we introduce the energy-momentum density, restoring the full set of hydrodynamic variables near the QCD critical point. We show that the relevant modes in the long-length, long-time limit are the same as in the universality class of the liquid-gas phase transition. We conclude with Sec. D. In Appendix A we show that the inclusion of the isospin density does not affect the critical behavior.

\section{HYDRODYNAMIC MODES NEAR THE QCD CRITICAL POINT}

Our analysis is based on the premise that at sufficiently large distance and time scales, the dynamics of a finite-temperature system is described by a hydrodynamic theory. It is a theory which contains only the degrees of freedom varying slowly with time. Although the validity of hydrodynamics is amply supported by experiments, a generic theoretical derivation, or proof, of the validity of such a description is a challenging problem. Nevertheless, the validity of hydrodynamic description has been verified in certain theories: scalar field theories [11] and strongly coupled gauge theories with gravity duals [12]. We shall therefore assume that the dynamics of the finite-temperature QCD plasma can be described by a set of hydrodynamic equations.

Generally, the full set of hydrodynamic modes include (i) densities of conserved charges, which relax via diffusion; (ii) phases of symmetry-breaking condensates; (iii) Abelian gauge fields of unbroken U(1) gauge symmetries. Near second-order phase transitions, (iv) the full order parameter (not only its phases, but also the magnitude) relax slowly, and therefore should be included in hydrodynamics. Thus, near the critical point of QCD on the $(T, \mu)$ plane, the modes potentially important for hydrodynamics are given by the fluctuations of:

- The conserved energy and momentum densities: $\varepsilon \equiv T^{00}-\left\langle T^{00}\right\rangle$, and $\pi^{i} \equiv T^{0 i}$;

- The conserved baryon number density, $n \equiv \bar{q} \gamma^{0} q-\left\langle\bar{q} \gamma^{0} q\right\rangle$;

- The chiral condensate $\sigma \equiv \bar{q} q-\langle\bar{q} q\rangle$.

The finiteness of the pion mass prevents pions from being a low-frequency hydrodynamic mode. This should be contrasted with the chiral limit, where the massless pions do become hydrodynamic modes, leading to a different universality class (both static and dynamic) 13].

In QCD, the complete set of conserved charge densities also includes isospin density (neglecting small isospin-breaking effects). However, the coupling of the isospin to other 
modes turns out to be irrelevant in the renormalization group sense (see Appendix A). Therefore we do not need to include the isospin density in our treatment.

\section{WITHOUT ENERGY-MOMENTUM TENSOR}

\section{A. Statics}

We start by discussing the statics. The static correlation functions can be found from a Ginzburg-Landau functional

$$
F[\sigma, n]=\int d \boldsymbol{x}\left[\frac{a}{2}\left(\partial_{i} \sigma\right)^{2}+b \partial_{i} \sigma \partial_{i} n+\frac{c}{2}\left(\partial_{i} n\right)^{2}+V(\sigma, n)\right]
$$

where

$$
V(\sigma, n)=\frac{A}{2} \sigma^{2}+B \sigma n+\frac{C}{2} n^{2}+\text { terms of higher orders. }
$$

The equilibrium distribution of the system is governed by $e^{-\beta F}$. The mixing between $\sigma$ and $n$ is not forbidden at nonzero baryon chemical potential and quark masses, and has been taken into account.

As we approach the phase transition, all the parameters of the Ginzburg-Landau functional $(a, b, c, A, B, C)$ remain finite, but the quadratic form in $V$ becomes degenerate, $A C=B^{2}$. At the phase transition, the potential has zero curvature along one direction in the $(\sigma, n)$ plane:

$$
\text { flat direction: } \quad \frac{\sigma}{n}=-\frac{B}{A}=-\frac{C}{B} \text {. }
$$

The response of the system to static external perturbation can be found by including source terms into the Ginzburg-Landau free energy. For example, changing the chemical potential from $\mu$ to $\mu+\delta \mu$ and/or the quark mass from $m_{\mathrm{q}}$ to $m_{\mathrm{q}}+\delta m_{\mathrm{q}}$ induces the following change in the average variables:

$$
\begin{aligned}
& \sigma=\Delta^{-1}\left(-B \delta \mu-C \delta m_{\mathrm{q}}\right), \\
& n=\Delta^{-1}\left(+A \delta \mu+B \delta m_{\mathrm{q}}\right) .
\end{aligned}
$$

where

$$
\Delta=A C-B^{2} .
$$

At the critical point $\Delta=0$ and the responses are singular. In particular, the baryon susceptibility $\chi_{\mathrm{B}} \equiv d n / d \mu$ diverges at the phase transition. Near the phase transition, both changes induced by $\delta \mu$ and $\delta m_{q}$ occur along the flat direction (3.3) of the potential.

From the distribution $e^{-\beta F}$ one can compute the fluctuations of $\sigma$ and $n$. In the limit $\boldsymbol{q} \rightarrow 0$ the quadratic fluctuations are

$$
\left\langle\sigma_{\boldsymbol{q} \rightarrow 0}^{2}\right\rangle=\frac{T C}{\Delta}, \quad\left\langle n_{\boldsymbol{q} \rightarrow 0}^{2}\right\rangle=T \chi_{B}=\frac{T A}{\Delta} .
$$

Finally, by considering the response to nonuniform perturbations, $\boldsymbol{q} \neq 0$, one can determine the correlation length $\xi$. Near the critical point it diverges as

$$
\xi \sim \Delta^{-1 / 2}
$$




\section{B. Hydrodynamic equations}

At the linear order, the hydrodynamic equations can be written by using the standard rules reviewed in, e.g., Ref. [10] (see also Ref. [14]). We define the variables conjugate to $\sigma$ and $n$ :

$$
\begin{aligned}
& X_{\sigma}=\frac{\delta F}{\delta \sigma}=\left(A-a \nabla^{2}\right) \sigma+\left(B-b \nabla^{2}\right) n, \\
& X_{n}=\frac{\delta F}{\delta n}=\left(B-b \nabla^{2}\right) \sigma+\left(C-c \nabla^{2}\right) n .
\end{aligned}
$$

The linearized equations for $\sigma$ and $n$ are then

$$
\begin{aligned}
& \dot{\sigma}(\boldsymbol{q})=-\gamma_{\sigma \sigma}(\boldsymbol{q}) X_{\sigma}(\boldsymbol{q})-\gamma_{\sigma n}(\boldsymbol{q}) X_{n}(\boldsymbol{q})+\xi_{\sigma}(\boldsymbol{q}), \\
& \dot{n}(\boldsymbol{q})=-\gamma_{n \sigma}(\boldsymbol{q}) X_{\sigma}(\boldsymbol{q})-\gamma_{n n}(\boldsymbol{q}) X_{n}(\boldsymbol{q})+\xi_{n}(\boldsymbol{q}) .
\end{aligned}
$$

We have written the equations for each spatial momentum $\boldsymbol{q}$. Onsager's principle forces $\gamma_{\sigma n}=\gamma_{n \sigma}$. The noise correlators are

$$
\left\langle\xi_{i}(t, \boldsymbol{q}) \xi_{j}\left(t^{\prime}, \boldsymbol{q}^{\prime}\right)\right\rangle=2 T \gamma_{i j}(\boldsymbol{q})(2 \pi)^{3} \delta\left(\boldsymbol{q}-\boldsymbol{q}^{\prime}\right) \delta\left(t-t^{\prime}\right), \quad i, j=\sigma, n,
$$

and are such so that the equilibrium distribution is given by $e^{-\beta F}$.

In the limit of small momenta $\boldsymbol{q} \rightarrow 0$ relevant to hydrodynamics, one can expand $\gamma_{i j}(\boldsymbol{q})$ in powers of $q^{2}$ and keep only the leading terms. Due to the conservation of baryon charge, $\gamma_{n \sigma}$ and $\gamma_{n n}$ vanish in the limit $\boldsymbol{q}=0$ and their expansions start at the order $q^{2}$. There is no such constraint on $\gamma_{\sigma \sigma}$, so the expansion for this coefficient starts at the $q^{0}$ order. Introducing the notations

$$
\begin{aligned}
& \gamma_{\sigma \sigma}(\boldsymbol{q})=\Gamma+O\left(q^{2}\right), \\
& \gamma_{\sigma n}(\boldsymbol{q})=\tilde{\lambda} q^{2}+O\left(q^{4}\right), \\
& \gamma_{n n}(\boldsymbol{q})=\lambda q^{2}+O\left(q^{4}\right),
\end{aligned}
$$

the hydrodynamic equations become

$$
\begin{aligned}
& \dot{\sigma}=-\Gamma \frac{\delta F}{\delta \sigma}+\tilde{\lambda} \nabla^{2} \frac{\delta F}{\delta n}+\xi_{\sigma}, \\
& \dot{n}=\tilde{\lambda} \nabla^{2} \frac{\delta F}{\delta \sigma}+\lambda \nabla^{2} \frac{\delta F}{\delta n}+\xi_{n} .
\end{aligned}
$$

with the noise correlators

$$
\begin{aligned}
& \left\langle\xi_{\sigma}(x) \xi_{\sigma}(y)\right\rangle=2 T \Gamma \delta^{4}(x-y), \\
& \left\langle\xi_{\sigma}(x) \xi_{n}(y)\right\rangle=-2 T \tilde{\lambda} \delta\left(t-t^{\prime}\right) \nabla^{2} \delta^{3}(\boldsymbol{x}-\boldsymbol{y}), \\
& \left\langle\xi_{n}(x) \xi_{n}(y)\right\rangle=-2 T \lambda \delta\left(t-t^{\prime}\right) \boldsymbol{\nabla}^{2} \delta^{3}(\boldsymbol{x}-\boldsymbol{y})
\end{aligned}
$$




\section{Modes}

Now let us insert the expression (3.1) for $F$ into the hydrodynamic equations and find the dispersion relations for the normal modes. To leading order in the limit $\boldsymbol{q} \rightarrow 0$ we find

$$
\begin{aligned}
& \dot{\sigma}=-\Gamma A \sigma-\Gamma B n, \\
& \dot{n}=(\tilde{\lambda} A+\lambda B) \nabla^{2} \sigma+(\tilde{\lambda} B+\lambda C) \nabla^{2} n .
\end{aligned}
$$

The eigenfrequencies of the hydrodynamic equations are found by solving the equation

$$
\operatorname{det}\left|\begin{array}{cc}
\Gamma A-i \omega & \Gamma B \\
(\tilde{\lambda} A+\lambda B) q^{2} & (\tilde{\lambda} B+\lambda C) q^{2}-i \omega
\end{array}\right|=0 .
$$

Near the critical point the two eigenfrequencies are

$$
\begin{aligned}
\omega_{1} & =-i \lambda \frac{\Delta}{A} \boldsymbol{q}^{2} . \\
\omega_{2} & =-i \Gamma A .
\end{aligned}
$$

Therefore, for small $\boldsymbol{q}$ there are two frequency scales: a small scale $\propto q^{2}$ and a larger scale $\propto q^{0}$. In the limit $q \rightarrow 0$ only $\omega_{1}$ is truly hydrodynamic. It is a diffusive mode with the diffusion constant

$$
D=\lambda \frac{\Delta}{A}=\lambda C-\lambda \frac{B^{2}}{A} .
$$

The diffusion constant $D$ tends to 0 as the critical point is approached, $\Delta \rightarrow 0$. To understand this fact consider $\Delta<0$. In this case the potential $V$ has an unstable direction. Negative $D$ means that, instead of relaxing, perturbations grow, leading to spinodal decomposition. The term $\lambda B^{2} / A$ in Eq. (3.17) can be interpreted as "antidiffusion" — at the critical point it exactly cancels out the normal diffusion term $\lambda C$.

To interpret the modes corresponding to $\omega_{1}$ and $\omega_{2}$, we find the eigenmodes of the hydrodynamic equations. Near $q=0$ the two eigenmodes are

$$
\left(\begin{array}{c}
-B \\
A
\end{array}\right) \text { and }\left(\begin{array}{l}
1 \\
0
\end{array}\right) \text {. }
$$

At the critical point, the first mode is the flat direction (3.3) of the potential energy $V$. Both $\sigma$ and $n$ vary along this direction. On the other hand, the mode with frequency $\omega_{2}$ corresponds to fluctuations of $\sigma$ alone, unaccompanied by any change of $n$.

The meaning of the two modes is simple. A generic smooth perturbation involving both $\sigma$ and $n$ will relax over two distinct time scales. First, at a much shorter time scale, $(\Gamma A)^{-1}$, the field $\sigma(\boldsymbol{x})$ alone adjusts to the values which at each point $\boldsymbol{x}$ in space minimize the potential $V(\sigma, n)$ at a given local value of $n(\boldsymbol{x}): \sigma=-(B / A) n$. After that, at longer time scales, the chiral order parameter $\sigma$ can be forgotten. It simply "traces" the profile of $n$, which relaxes to $n=0$ much slower, over the diffusive time scale $\left(D \boldsymbol{q}^{2}\right)^{-1}$. From the point of view of slow dynamics at long distances, the addition of the chiral order parameter $\sigma$ does not change the physics, which is exactly that of the liquid-gas phase transition, with a conserved density as the only hydrodynamic mode (besides the energy-momentum). 


\section{Real-time correlators}

From the stochastic equations one can compute the real-time correlators of $\sigma$ and $n$. Assuming we are in the regime $D q^{2} \ll \Gamma A$, the correlators can be written as

$$
\begin{aligned}
\left\langle\sigma_{\omega \boldsymbol{q}}^{2}\right\rangle & =\frac{2 T \Gamma}{\omega^{2}+\Gamma^{2} A^{2}}+\frac{2 T B^{2} \lambda q^{2}}{A^{2}\left(\omega^{2}+D^{2} q^{4}\right)}, \\
\left\langle n_{\omega \boldsymbol{q}}^{2}\right\rangle & =\frac{2 T \lambda q^{2}}{\omega^{2}+D^{2} q^{4}} .
\end{aligned}
$$

Integrating over $\omega$ we recover the equal-time correlators (3.6). Notice that while $\left\langle\sigma^{2}\right\rangle$ obtains contribution from two characteristic scales $\omega \sim \Gamma A$ and $\omega \sim D q^{2}$, the fluctuations of $n$ are peaked only at the latter scale.

\section{E. Baryon diffusion, susceptibility and critical indices in $d=3$}

We have seen that, from the point of view of real-time dynamics, the criticality is manifested by vanishing of the baryon diffusion rate $D$ given by Eq. (3.17). Comparing to (3.6), we find that

$$
D=\lambda \chi_{\mathrm{B}}^{-1}
$$

This relation has a simple physical explanation. Consider a configuration where $n$ varies with space, $n=n(\boldsymbol{x})$. This corresponds to a spatially varying chemical potential $\mu(\boldsymbol{x})=\chi_{\mathrm{B}}^{-1} n(\boldsymbol{x})$. Recall that $\mu$ acts as the time component $A_{0}$ of an external gauge field coupled to the baryon current. Thus such a chemical potential $\mu(\boldsymbol{x})$ corresponds to an external electric field $\boldsymbol{E}=-\boldsymbol{\nabla} \mu$ acting on the baryon charge. The baryon current induced by this field is

$\boldsymbol{j}_{n}=\lambda \boldsymbol{E}=-\lambda \chi_{\mathrm{B}}^{-1} \boldsymbol{\nabla} n$, where $\lambda$ is the baryon conductivity. Using the definition of diffusion rate $\boldsymbol{j}_{n}=-D \nabla n$ we obtain (3.20).

At $d=3$ one has to take into account fluctuations which will modify the numerical values of the critical exponents. With the energy-momentum density fluctuations frozen, as in this section, the universality class is that of model B in the Hohenberg-Halperin classification [8]. It is a model of the uniaxial ferromagnet — with a single conserved mode. In the model B the diffusion constant is still inversely proportional to the susceptibility, as in Eq. (3.20):

$$
D \sim \chi_{\mathrm{B}}^{-1} \sim \xi^{-2+\eta}
$$

but the divergence of $\chi_{B}$ is modified by $\eta \approx 0.04$ - a critical index of the $3 \mathrm{~d}$ Ising model, relative to the mean field result.

The dispersion relation $\omega=-i D q^{2}$ applies only in the regime $q \ll \xi^{-1}$. When $q \gg \xi^{-1}$, we are in the critical regime, and higher powers of $q$ are non-negligible. In this regime the scaling dictates dispersion $\omega \sim q^{z}$. The two behaviors should match smoothly at $q \sim \xi^{-1}$. From Eq. (3.21) one then finds $z=4-\eta$ (in mean-field theory $z=4$ ). 


\section{COUPLING TO ENERGY-MOMENTUM}

So far we have completely neglected the motion of the plasma, regarding the latter as a static medium. Now we will allow the plasma to move - this will modify the hydrodynamic equations and change the value of the dynamic critical exponent.

As we have learned in the previous Section, only one combination of $\sigma$ and $n$ is truly hydrodynamic. This mode can be thought of as the baryon charge density $n$, with $\sigma$ simply tracing $n$ and not independent. At sufficiently long timescales, the hydrodynamic theory contains this mode, the energy density $\varepsilon$ and the momentum density $\pi^{i}$. The linearized theory has four eigenmodes: the baryon diffusion mode, two diffusive transverse shear modes and a propagating longitudinal sound wave.

At very low momenta, and long time scales, sound wave mode can be effectively integrated out, since it has linear dispersion $\omega \sim q$ and its frequency is much higher than the frequencies of the remaining diffusive modes $\omega \sim q^{2}$. In other words, the fluctuations of the energy density and pressure caused by sound excitations are fast and average out - there are no sound waves on these long diffusive time scales. The number of remaining hydrodynamic modes is three: two transverse components of $\pi^{i}$ and the baryon density $n .^{1}$

The same set of modes describes hydrodynamics of the liquid-gas phase transition [15]. Here we only review the results of previous studies, referring the reader to the original literature [8, 15] for further details.

The liquid-gas phase transition belongs to the dynamic universality class of model $\mathrm{H}$. This model describes a system with a conserved order parameter, conserved and transverse momentum density, and nonzero Poisson bracket between the two. The same model describes the critical point of binary fluids. The hydrodynamic theory contains two kinetic coefficients: the shear viscosity $\bar{\eta}$ and the diffusion constant (in our case, that of the baryon charge $D$ ). ${ }^{2}$

Both kinetic coefficients are singular (nonanalytic) at the phase transition:

$$
\begin{aligned}
D=\lambda \chi_{\mathrm{B}}^{-1} & \sim \xi^{x_{\lambda}} \chi_{\mathrm{B}}^{-1}, \\
\bar{\eta} & \sim \xi^{x_{\eta}} .
\end{aligned}
$$

The two new dynamical critical exponents satisfy the following relation

$$
x_{\lambda}+x_{\eta}=4-d-\eta \text {. }
$$

The enhancement of $D$ by a power of $\xi$ is due to the contribution of convection to the baryon conductivity $\lambda$. Although the calculation of $x_{\lambda}$ and $x_{\eta}$ individually is not simple, the relation (4.2) has a simple physical explanation [8]. Let us apply the field $\boldsymbol{E}=-\boldsymbol{\nabla} \mu$

\footnotetext{
${ }^{1}$ The physically intuitive argument presented here is corroborated by the analysis of linear mixing of four variables: $\sigma, n, \epsilon$ and the longitudinal component of $\pi^{i}$. As in Section [III there is only one diffusive mode, in which $\sigma$ and $\epsilon$ trace the local value of $n$ to minimize the local value of the thermodynamic potential (i.e., maximize the pressure).

${ }^{2}$ We choose the notation $\bar{\eta}$ for the shear viscosity to avoid confusion with the static critical exponent $\eta$. The constant of heat conductance $\kappa$ is not an independent constant, but is related to $D$ by $D \propto \kappa \chi_{\mathrm{B}}^{-1}$.
} 
introduced already in Section III. In model B, without convection, this field would induce baryon current via diffusion: $\boldsymbol{j}_{n}=\lambda \boldsymbol{E}$, where $\lambda$ is finite. However, in this field $\boldsymbol{E}$, the baryon charge carrying fluid will experience mechanical force equal to $n \boldsymbol{E}$ per unit volume. The fluid will accelerate to velocity $\boldsymbol{v}$ at which viscous drag balances the external force: $\boldsymbol{f}_{\text {visc }}+\boldsymbol{f}_{\text {appl }}=0$. For a chunk of fluid of typical linear dimension $L$ the drag and the applied forces are of order:

$$
\boldsymbol{f}_{\mathrm{visc}} \sim-\bar{\eta} \rho \boldsymbol{v} L^{d-2}, \quad \text { and } \quad \boldsymbol{f}_{\mathrm{appl}} \sim n \boldsymbol{E} L^{d},
$$

where $\rho$ is the mass density. The corresponding velocity $\boldsymbol{v}$ diverges with $L$ and so does the induced baryon current:

$$
\boldsymbol{j}_{n}=n \boldsymbol{v} \sim \frac{n^{2}}{\bar{\eta} \rho} L^{2} \boldsymbol{E} .
$$

The divergence is cut off at the scale $L \sim \xi$ because the baryon number fluctuation, given by $\left\langle n^{2}\right\rangle=T \chi_{\mathrm{B}} / L^{d}$ for $L \gg \xi$, is correlated on at most that scale. Therefore at the critical point we find a singular contribution to the product of the kinetic coefficients

$$
\lambda \bar{\eta} \sim\left\langle n^{2}\right\rangle \xi^{2} \sim \chi_{\mathrm{B}} \xi^{2-d} \sim \xi^{4-d-\eta}
$$

which leads to Eq. (4.2).

In the $d=3$ Ising model $\eta$ is very small. It has been found [8, 15, 16] that the divergence of the shear viscosity is very weak, or $x_{\eta}$ is numerically small. According to Eq. (4.2), therefore, $x_{\lambda} \approx 1$. Calculations using renormalization group in $4-\epsilon$ dimensions yields the values [8, 15]

$$
\begin{aligned}
& x_{\eta}=\frac{1}{19} \epsilon(1+0.238 \epsilon+\cdots) \approx 0.065, \\
& x_{\lambda}=\frac{18}{19} \epsilon(1-0.033 \epsilon+\cdots) \approx 0.916,
\end{aligned}
$$

while mode-coupling calculations done directly in $d=3$ and assuming $\eta=0$ yield [16]

$$
x_{\eta}=0.054, \quad x_{\lambda}=0.946 .
$$

One sees that in model $\mathrm{H}$ the diffusion constant still vanishes at the critical point:

$$
D \sim \xi^{-2+\eta+x_{\lambda}}
$$

but the power is closer to 1 rather than to 2 as in model B. That is due, as we have mentioned, to fluctuations of the fluid motion. Using the same argument as the one presented at the end of Sec. III, we find

$$
z=4-\eta-x_{\lambda} \approx 3
$$

\section{CONCLUSION}

We have seen that the dynamic universality class of the QCD critical point is that of model H, i.e., the liquid-gas phase transition. The chiral order parameter, being non-conserved and mixing with the conserved baryon charge, does not affect the dynamic universality class. 
Here we would also like to compare our results to the universality class argument of Ref. 7]. The argument is based on three assumptions, spelled in Ref. [7]: i) the chiral order parameter $\sigma$ is not conserved; ii) there are other conserved quantities, such as the baryon density $n$; iii) the Poisson brackets between $\sigma$ and the conserved quantities vanish. While the first two assumptions are correct, the last assumption is not. There is a non-zero Poisson bracket between $\sigma$ (or $n$ ) and the momentum density $\left[\pi_{i}(\boldsymbol{x}), \sigma(\boldsymbol{y})\right]=\sigma(\boldsymbol{x}) \nabla_{i} \delta^{3}(\boldsymbol{x}-\boldsymbol{y})$. As we have seen, the coupling to momentum density plays essential role in determining the dynamic universality class. Another, more subtle implicit assumption of Ref. [7] is that the order parameter $\sigma$ and baryon density $n$ cannot mix. This assumption leads to model C, where the mixing is forbidden by a symmetry of the order parameter (it would be $\sigma \rightarrow-\sigma$ in this case). As we have seen in Section III the $\sigma n$ mixing eliminates nonconserved mode from hydrodynamic theory, leading instead to model B.

The model $\mathrm{H}$ value of $z \approx 3$ is larger than the model $\mathrm{C}$ value $z \approx 2.17$ used in Ref. [7]. As discussed in the introduction, this means that the effect of the time constraint on the correlation length $\xi$ is stronger. Thus the numerical estimate of maximal $\xi$ in Ref. 7] should be revised downward. However, we do not expect the main conclusions of Ref. [7] to change qualitatively. ${ }^{3}$

An interesting step beyond the relaxation equation for the diverging correlation length in Ref. [7], which one can contemplate, having the correct hydrodynamic theory in hand, is to consider real-time evolution of the hydrodynamic modes near criticality, similar to the study of Ref. [18].

The pions do not enter the hydrodynamic theory as long as the correlation length $\xi$ is sufficiently large compared to the inverse of the pion mass $1 / m_{\pi}$ (see Section II). Although this condition is always fulfilled sufficiently close to the critical point, in the realistic case of a heavy ion collision, the maximal achievable $\xi(2-3 \mathrm{fm}$, according to [6, 7] $)$ is not significantly larger than $1 / m_{\pi}$. Therefore it might be interesting to study the effects due to the crossover between the two regimes $\xi \gg 1 / m_{\pi}($ model $\mathrm{H})$ and $\xi \ll 1 / m_{\pi}(\mathrm{O}(4)$ antiferromagnet [13]).

It would be interesting to explore the phenomenological consequences of the vanishing baryon diffusion rate for heavy ion collisions. Most likely it will manifest itself in the fluctuations of the baryon number [19].

\section{Acknowledgments}

We thank H. Fujii and K. Rajagopal for discussions and for their comments on the manuscript. M.A.S. thanks RIKEN BNL Center and U.S. Department of Energy [DEAC02-98CH10886] for providing facilities essential for the completion of this work. D.T.S. is supported, in part, by DOE grant No. DOE-ER-41132 and the Alfred P. Sloan Foundation. M.A.S. is supported, in part, by DOE grant No. DE-FG0201ER41195 and by the Alfred P.

\footnotetext{
${ }^{3}$ Rerunning the codes of Ref. 7] with the new value of $z$ shows [17] that the required revision is numerically small (less than 10\%) and is within the many uncertainties of the method detailed in Ref. 7]. We thank Krishna Rajagopal for sharing this result with us.
} 
Sloan Foundation.

\section{APPENDIX A: ISOSPIN DENSITY AND SCALING DIMENSION COUNTING}

In this appendix we show that the inclusion of the isospin density $n_{\mathrm{I}}$ does not affect the critical behavior. For simplicity, let us neglect the energy-momentum tensor and discuss only the coupled system of the isospin density and the baryon density. The coupled dynamics at the critical point is given by a pair of stochastic equations:

$$
\begin{aligned}
& \dot{n}_{\mathrm{B}}=\lambda_{\mathrm{B}} \nabla^{2}\left(-c \boldsymbol{\nabla}^{2} n_{\mathrm{B}}+g_{\mathrm{B}} n_{\mathrm{B}}^{3}+\frac{g_{\mathrm{BII}}}{2} n_{\mathrm{I}}^{2}\right)+\xi_{\mathrm{B}} ; \\
& \dot{n}_{\mathrm{I}}=\lambda_{\mathrm{I}} \nabla^{2}\left(\chi_{\mathrm{I}}^{-1} n_{\mathrm{I}}+g_{\mathrm{I}} n_{\mathrm{I}}^{3}+g_{\mathrm{BII}} n_{\mathrm{B}} n_{\mathrm{I}}\right)+\xi_{\mathrm{I}} .
\end{aligned}
$$

Note that the term $\lambda_{\mathrm{B}} \chi_{\mathrm{B}}^{-1} \nabla^{2} n_{\mathrm{B}}$ in Eq. (A1a) vanishes at the critical point, because $\chi_{\mathrm{B}} \rightarrow \infty$. The isospin susceptibility $\chi_{\mathrm{I}}$ is finite [19]. We kept only coupling terms of lowest order (most relevant in the infrared) consistent with the symmetry $n_{\mathrm{I}} \rightarrow-n_{\mathrm{I}}$. The noises $\xi_{\mathrm{B}}$ and $\xi_{\mathrm{I}}$ are auto-correlated as

$$
\begin{aligned}
\left\langle\xi_{\mathrm{B}}(t, \boldsymbol{x}) \xi_{\mathrm{B}}\left(t^{\prime}, \boldsymbol{y}\right)\right\rangle & =-2 T \lambda_{\mathrm{B}} \delta\left(t-t^{\prime}\right) \nabla^{2} \delta^{d}(\boldsymbol{x}-\boldsymbol{y}) \\
\left\langle\xi_{\mathrm{I}}(t, \boldsymbol{x}) \xi_{\mathrm{I}}\left(t^{\prime}, \boldsymbol{y}\right)\right\rangle & =-2 T \lambda_{\mathrm{I}} \delta\left(t-t^{\prime}\right) \nabla^{2} \delta^{d}(\boldsymbol{x}-\boldsymbol{y}) .
\end{aligned}
$$

Here $d$ is the number of spatial dimensions.

The canonical scaling dimensions involved in renormalization group transformations are determined as follows. Comparing linear terms in Eq. A1a we establish that if space has dimension $-1,[x]=-1$, then the dimension of time is $[t]=-4$. The dimension of noises are then found from Eqs. (A2) to be $\left[\xi_{\mathrm{B}}\right]=\left[\xi_{\mathrm{I}}\right]=d / 2+3$. From Eq. (A1a) one then finds $\left[n_{\mathrm{B}}\right]=d / 2-1$. In Eq. (A1b we see that the term $\dot{n}_{\mathrm{I}}$ can be neglected in the infrared, so $\left[n_{\mathrm{I}}\right]=d / 2+1$.

Using these scaling dimensions for the fields, we can now determine the scaling dimensions of the coupling terms. One finds that $\left[\lambda_{\mathrm{B}} g_{\mathrm{BII}}\right]=-1-d / 2$, and $\left[\lambda_{\mathrm{I}} g_{\mathrm{BII}}\right]=1-d / 2$, so the coupling between baryon and isospin densities is irrelevant for $d>2$. (In contrast, $\left[\lambda_{\mathrm{B}} g_{\mathrm{B}}\right]=4-d$, so the $n_{\mathrm{B}}^{3}$ term is relevant for $d<4$.)

Similarly one can show that isospin density remains decoupled from the critical dynamics when the energy-momentum tensor is included. This simple power counting scheme can be formalized by rewriting the stochastic equations as a path integral.

[1] For a review, see, e.g., K. Rajagopal and F. Wilczek, "The condensed matter physics of QCD," hep-ph/0011333.

[2] P. Braun-Munzinger, K. Redlich and J. Stachel, nucl-th/0304013.

[3] M. Asakawa and K. Yazaki, Nucl. Phys. A 504, 668 (1989);

A. Barducci, R. Casalbuoni, G. Pettini and R. Gatto, Phys. Rev. D 49, 426 (1994);

J. Berges and K. Rajagopal, Nucl. Phys. B 538, 215 (1999) hep-ph/9804233; 
M. A. Halasz, A. D. Jackson, R. E. Shrock, M. A. Stephanov and J. J. M. Verbaarschot, Phys. Rev. D 58, 096007 (1998) hep-ph/9804290;

O. Scavenius, A. Mocsy, I. N. Mishustin and D. H. Rischke, Phys. Rev. C 64, 045202 (2001) nucl-th/0007030;

Y. Hatta and T. Ikeda, Phys. Rev. D 67, 014028 (2003) hep-ph/0210284;

N. G. Antoniou and A. S. Kapoyannis, Phys. Lett. B 563, 165 (2003) hep-ph/0211392.

[4] Z. Fodor and S. D. Katz, J. High Energy Phys. 0203, 014 (2002) hep-lat/0106002;

P. de Forcrand and O. Philipsen, Nucl. Phys. Proc. Suppl. 129, 521 (2004) hep-lat/0309109;

F. Karsch, C. R. Allton, S. Ejiri, S. J. Hands, O. Kaczmarek, E. Laermann, and C. Schmidt, Nucl. Phys. Proc. Suppl. 129, 614 (2004) hep-lat/0309116.

[5] M. A. Stephanov, K. Rajagopal, and E. V. Shuryak, Phys. Rev. Lett. 81, 4816 (1998) hep-ph/9806219.

[6] M. A. Stephanov, K. Rajagopal, and E. V. Shuryak, Phys. Rev. D 60, 114028 (1999) hep-ph/9903292.

[7] B. Berdnikov and K. Rajagopal, Phys. Rev. D 61, 105017 (2000) hep-ph/9912274.

[8] P. C. Hohenberg and B. I. Halperin, Rev. Mod. Phys. 49, 435 (1977).

[9] H. Fujii, Phys. Rev. D 67, 094018 (2003) hep-ph/0302167;

H. Fujii and M. Ohtani, hep-ph/0401028.

[10] L. D. Landau and E. M. Lifshitz, Statistical Physics part 1 (Pergamon Press, 1980).

[11] S. Jeon, Phys. Rev. D 52, 3591 (1995) hep-ph/9409250;

S. Jeon and L. G. Yaffe, Phys. Rev. D 53, 5799 (1996) hep-ph/9512263.

[12] G. Policastro, D. T. Son, and A. O. Starinets, J. High Energy Phys. 0209, 043 (2002) hep-th/0205052.

[13] K. Rajagopal and F. Wilczek, Nucl. Phys. B 399, 395 (1993) hep-ph/9210253.

[14] D. T. Son and M. A. Stephanov, Phys. Rev. D 66, 076011 (2002) hep-ph/0204226.

[15] E. D. Siggia, B. I. Halperin, and P. C. Hohenberg, Phys. Rev. B 13, 2110 (1976).

[16] T. Ohta and K. Kawasaki, Prog. Theor. Phys. 55, 1384 (1976).

[17] K. Rajagopal, private communication.

[18] L. M. A. Bettencourt, K. Rajagopal, and J. V. Steele, Nucl. Phys. A 693, 825 (2001) hep-ph/0106257.

[19] Y. Hatta and M. A. Stephanov, Phys. Rev. Lett. 91, 102003 (2003) [Erratum-ibid. 91, 129901 (2003)] hep-ph/0302002. 